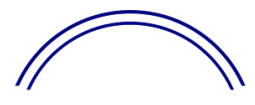

\title{
Intimate Partner Violence Among Pregnant Saudi Women: Prevalence, Risk Factors, and Attitudes
}

\author{
Hatoon A. Al Taifi, ${ }^{1}$ Abdullah H. Alqahtani, ${ }^{1,2}$ Nourah H. Al Qahtani ${ }^{1}$ \\ ${ }^{1}$ Imam Abdulrahman Bin Faisal University, Dammam, Saudi Arabia; ${ }^{2}$ Michael G. DeGroote School of Medicine, \\ McMaster University, Hamilton, Canada
}

\begin{abstract}
Intimate partner violence (IPV) during pregnancy has become a focus of attention in recent years, owing to its relatively high prevalence, its impact on maternal and fetal health, and its cumulative effects over time. This study aims to determine the magnitude and characteristics of IPV among pregnant Saudi Arabian women, to identify the factors that increase the risk, and to assess the willingness of abused women to report IPV. This is a cross-sectional, community-based survey of pregnant women in the Eastern Province of Saudi Arabia. A modified Abuse Assessment Score (AAS) questionnaire was used. Sociodemographic data were collected. Data related to willingness to report IPV, including reasons for declining to report IPV, were also collected. A total of 1,330 women completed the three parts of the survey. In total, $345(25.9 \%)$ women reported emotional abuse during pregnancy, whereas $72(5.4 \%)$ reported physical abuse and $180(13.5 \%)$ reported sexual abuse. In emotional abuse, a significant association was found between having more children $(p=.001)$, having a lower education $(p=.05)$, having a lower income $(p=.04)$, and being abused. In physical abuse during pregnancy, no significant associations were found between all variables and being abused. However, in reporting sexual abuse among women during pregnancy, a significant increase in the risk was found in those with four or more children $(p=.01)$ and those who are employed $(p=.01)$. More than two-thirds $(71.2 \%)$ of abused pregnant women were unwilling to report the abusive acts to a medical authority. IPV is common among pregnant Saudi women. Emotional abuse is the commonest form of IPV, affecting one in four women. More than two-thirds of abused women were unwilling to report their partner's abusive acts. Screening for IPV may encourage women to seek help and improve both maternal and fetal health.
\end{abstract}

Key words: Intimate partner violence, Saudi Arabia, pregnancy and abuse, Abuse Assessment Score, emotional abuse, physical abuse, sexual abuse

\section{Introduction}

Intimate partner violence (IPV) during pregnancy has become a focus of attention in recent years, owing to its relatively high prevalence, its impact on maternal and fetal health, and its cumulative effects over time. According to the World Health Organization (WHO) [1], IPV is manifested by behaviours that cause physical, sexual, or psychological harm, such as acts of physical aggression, sexual coercion, psychological abuse, and controlling behaviours. The Family Violence Prevention Fund [2], gives a broader definition: "Intimate partner violence is a pattern of assaultive and coercive behaviors that may include inflicted physical injury, psychological abuse, sexual assault, progressive isolation, stalking, deprivation, intimidation, and threats." 
Comparing IPV in pregnancy to medical diseases for which pregnant women are routinely screened, such as gestational diabetes and pre-eclampsia, IPV may be as common or even more common than those conditions, with measurable effects on maternal and fetal health $[3,4]$. Routine screening for IPV during pregnancy is a matter of debate, as most abused women tend not to disclose abusive acts. Multiple systematic reviews of IPV screening programs have provided insufficient data from which to recommend for or against routine screening [5]. However, many groups recommend routine screening, including the American College of Obstetricians and Gynecologists, who advised in 2012 that all women be screened for IPV at periodic intervals, in the first prenatal visit, at least once per trimester, and at the postpartum checkup [6]. In contrast, the WHO [1] issued guidelines that advised against the routine screening for IPV in women. However, they recommended that women who have a significantly increased risk, or who present with signs or symptoms suggesting possible IPV (e.g., unexplained injuries or depression), be asked about possible IPV exposure.

Nelson et al. [7] systematically reviewed the effectiveness of screening and interventions in reducing IPV and related health outcomes, as well as the accuracy of screening tools. They concluded that screening could reduce IPV and improve health outcomes, and that the screening instruments designed for healthcare settings could accurately identify women experiencing IPV.

Kiely et al. [8] conducted a randomized controlled trial of 1,044 pregnant African-American women to evaluate the efficacy of a psycho-behavioural intervention during prenatal and postpartum care on the reduction of IPV recurrence and improved pregnancy outcomes. They compared usual care to perinatal counselling in addressing IPV-related danger. The trial showed efficacy in reducing IPV victimization and improving pregnancy outcomes, with decreased rates in recurrent IPV and depression.
Violence against pregnant women carries a high risk of morbidity and mortality. This risk includes mental health issues as abused pregnant women are more likely to develop psychological disorders, have an increased risk of depression and suicide attempts, and have a higher risk for substance abuse and sexual dysfunction [5]. In addition, abused pregnant women are at greater risk of further abuse, and in severe danger of murder [9]. Desmarais et al. [10] also found a relation between abuse during pregnancy and postpartum mental health, as these women were more liable to develop posttraumatic stress disorder, depression, and obsessive-compulsive disorder. Physical injuries in abused women ranged from $40 \%$ to $72 \%$ [11].

During pregnancy, maternal complications of IPV include miscarriages, infections, low weight gain, antepartum hemorrhage, preterm labour and birth, premature rupture of membrane, and maternal death $[5,11,12]$. Maternal mortality rates were reported to be as high as $16 \%$ in one study conducted in India, which makes it the second most common cause of maternal death, after postpartum hemorrhage. However, the authors believe that the prevalence could be higher, owing to underreported and misclassified maternal deaths [13].

Fetal complications of IPV include intrauterine growth restriction, low birth weight, fetal injury, and death $[5,14]$. Perinatal and neonatal death rates were also increased, mostly owing to prematurity $[15,16]$.

Data conflicts as to whether pregnancy is considered a risk factor for violence initiation or escalation, or if pregnancy is a protective factor, most likely owing to differences in research design and assessment. Castro et al. [17] compared the prevalence of emotional, physical, and sexual abuse before and during pregnancy, and found no statistically significant differences in the prevalence. In addition, in a longitudinal review of data, Jasinski [18] found that pregnant women were no more or less likely to be abused than non-pregnant women. 


\section{Prevalence}

The problem with estimating prevalence among pregnant women is the use of different assessment tools, which give different results. Using questionnaires with general non-specific questions about abuse has led to the underestimation of the prevalence, compared with using more behaviourally specific questions. Moreover, personal interviews, using a standardized, psychometrically sound measure of physical violence, resulted in higher rates of reporting of violence than self-reported, singleitem measures [19].

Prevalence of IPV during pregnancy ranged from $6 \%$ to $15 \%$ in population-based studies done in Canada, Chile, Egypt, and Nicaragua [11]. In an American study, the estimated prevalence of IPV during pregnancy ranged between 3.9\% and $8.3 \%$ [3]. The WHO multi-country study [20], which screened 24,000 women from 10 different countries (it did not include Saudi Arabia), reported a lifetime prevalence of physical and (or) sexual IPV to be between $15 \%$ and $71 \%$. In developed countries, Gazmararian et al. [4] concluded that the prevalence of IPV ranged from $0.9 \%$ to $20.0 \%$. These major differences in prevalence were thought to be due to different methods of screening and to differences in the populations studied. Kashif et al. [21] conducted a systematic, integrated review of literature from Bangladesh, Pakistan, India, China, Thailand, and Iran. They found the prevalence of violence during pregnancy ranged between $4.3 \%$ and $48.0 \%$. In a developing country such as Zimbabwe, Shamu et al. [22] estimated that $63.1 \%$ of pregnant women reported physical, emotional, and (or) sexual IPV during pregnancy, with $10.0 \%$ of them reporting severe violence during pregnancy.

These high rates of prevalence during pregnancy are thought to be inaccurate, as most abused women are reluctant to disclose IPV, owing to different psychological and social factors [14].

To our knowledge, there is no estimation of the prevalence of IPV among pregnant Saudi women. It is assumed that the IPV rate is high in an Arabic country, owing to cultural and environmental reasons. In Arabic communities, the victim may be blamed for angering her husband. IPV is sometimes seen as a justifiable response to misbehaviour and wrongdoing of the wife. Arabic women tend to consider the abuse as a private family issue and believe that the family should be the first help-seeking option [23].

This study aims to determine the magnitude, scope, and characteristics of IPV, to identify the factors that increase the risk, and to assess the willingness of abused women to report IPV. Knowing the prevalence will help Arabic communities in acknowledging the existence of IPV, including knowing the reasons that are preventing women from seeking help. It will also provide guidance to these communities in the development of programs to reach out to victims of IPV.

\section{Methods}

This cross-sectional, community-based survey of pregnant Saudi women was approved by the Institutional Review Board of Imam Abdulrahman Bin Faisal University. A modified Abuse Assessment Score (AAS) questionnaire was used. The study was conducted in the Eastern Province of Saudi Arabia, from January to June 2016. The questionnaires were given to pregnant women visiting antenatal clinics in hospitals and primary health-care centres across the Eastern Province (in the following cities: Khobar, Dammam, Qatif, Huffof, and Jubail). Inclusion criteria were as follows: women with a current pregnancy of any gestational age, Muslim Arabic ethnicity, and currently married.

The questionnaire consisted of three parts. The first part covered the sociodemographic characteristics of the participants, including age, number of children, educational level, family income, employment status, and living arrangement.

The second part of the questionnaire was the assessment tool. IPV was measured using four modified and translated questions of the AAS 
questionnaire. The first question asked about emotional abuse during the pregnancy (Have you ever been emotionally abused by your husband in the form of verbal abuse, intimidation, or ridicule during your pregnancy?). The second question was about physical abuse in the year before her current pregnancy (Within the last year, have you been hit, slapped, kicked, or otherwise physically hurt by your husband?). The third question was about physical abuse during this pregnancy (Since you have been pregnant, have you been hit, slapped, kicked, or otherwise physically hurt by your husband?). The fourth question was about sexual abuse in pregnancy (During your pregnancy, has anyone forced you to have any form of sexual activities?). In all of the questions used, the husband was referred to as the abuser. Answering positively to any of these questions would identify the woman as being abused.

The third part of the questionnaire included questions developed and added by the researchers about how willing the participants were to report the abuse and the reasons for not reporting. Abused women were asked a question about their willingness to seek help in the presence of a competent medical authority. Participants who did not want to seek help were asked about the reasons that prevent them from seeking help.

Respondents were asked whether they believed that the abuse by their husband was justifiable because of their (perceived) failure in their duties toward him or because the husband was under psychological stresses that might explain and justify his abuse. In addition, they were asked if they thought this abusive act was an isolated event. Respondents were asked if they were not reporting the assault because they thought it was a private matter that must be resolved internally, without seeking help outside the family framework, or if they thought that reporting the issue would expose them to more abuse. In addition, they were asked if they were not reporting the assault because of their fear that seeking help would lead to separation or divorce, and thus the disintegration of the family. In addition, respondents were asked if they believed that the assault was justifiable, owing to religious reasons. An example of a religious reason would be that Muslim women believe that being patient and obedient to their husband will be rewarded. Finally, respondents were asked if they believed that reporting the assault would not help them.

A total of 1,559 pregnant women answered the questionnaire; among these, 229 were excluded, because they had not completed all of the questions. A total of 1,330 women completed the three parts of the questionnaire and were included in the analysis.

Sample size was calculated based on a precision of $4 \%$ and a confidence interval of $99 \%$. The estimated population was entered as 32,000 , which is the total number of deliveries in the Eastern Province. The estimated prevalence, based on previous studies, was $50 \%$. The following formula was used:

$$
n=(t)^{2} \times p(1-p) / m^{2}
$$

The required sample size was 1,005 . An additional sample of $20 \%(n=201)$ was added to make up for lost or incomplete data. The total size sample was estimated to be 1,206.

Data were analyzed using the Statistical Analysis Package for the Social Sciences, version 23.0 (IBM Corp., Armonk, NY, USA). Data were presented as frequencies and percentages. Differences between the two groups were analyzed by using the chi-square test. A $p<.05$ was considered significant.

\section{Results}

A total of 1,330 women were included in the survey. Their ages ranged from 14 to 50 years, with a mean age of 29.8 years (standard deviation 6.57 years). Most women were in their twenties, university-educated, unemployed housewives, with an average-to-high family income, less than four children, and lived with their husband in a separate house (not with their spouse's family). The sociodemographic characteristics of all the participants are shown in Table 1. 
Table 1: Sociodemographic characteristics of survey participants $(N=1,330)$

\begin{tabular}{lc}
\hline Variable & $\mathrm{n}(\%)$ \\
\hline Age, years & \\
$14-19$ & $32(2.4)$ \\
$20-29$ & $660(49.6)$ \\
$30-39$ & $512(38.5)$ \\
$40-50$ & $126(9.5)$ \\
Number of children & \\
Zero & $254(19.1)$ \\
$1-3$ & $720(54.1)$ \\
$4-10$ & $356(26.8)$ \\
Education & \\
Illiterate & $6(0.5)$ \\
Elementary/middle school & $101(7.6)$ \\
High school & $427(32.1)$ \\
Diploma & $57(4.3)$ \\
University & $711(53.5)$ \\
Masters & $16(1.2)$ \\
Doctorate & $12(0.9)$ \\
Family income, Saudi riyal/month & \\
<2000 & $124(9.3)$ \\
2000-8000 & $601(45.2)$ \\
>8000 & $605(45.5)$ \\
Employment & \\
Employed & \\
Unemployed & \\
Living arrangement & \\
With spouse & \\
With spouse's family & \\
\hline
\end{tabular}

Among the 1,330 women in the study sample, $345(25.9 \%)$ were emotionally abused during pregnancy, $72(5.4 \%)$ reported physical abuse during pregnancy, and $180(13.5 \%)$ reported sexual abuse during pregnancy.

Differences between women who reported emotional, physical, or sexual abuse during pregnancy and those who did not are presented in Table 2. As shown, age and living arrangement were not significantly associated with any type of abuse during pregnancy. In emotional abuse, there was a significant association between having more children $(p=$
$.001)$, lower education ( $p=.05)$, lower income $(p=.04)$, and being abused. In physical abuse during pregnancy, no significant associations were found between the sociodemographic variables and being abused. However, in reporting sexual abuse during pregnancy, a significant increase in risk was found in women with four or more children $(p=.01)$ and those who were employed $(p=.01)$.

Table 3 shows the different sociodemographic variables in relation to women who reported any type of abuse in the total number of participants, women who reported any type of abuse during pregnancy only, and women who reported physical abuse before the pregnancy. Among the 1,330 women, 420 (31.57\%) women reported being abused, either during their current pregnancy or in the year preceding their pregnancy. Having four or more children $(p=$ $.001)$ and having a lower education $(p=.01)$ appeared as significant risk factors. Similarly, having four or more children $(p=.001)$ and lower education $(p=.01)$ appeared as significant risk factors for being abused during pregnancy, with a total of $410(30.83 \%)$ participants reporting any type of abuse during pregnancy.

A total of 121 women reported physical abuse in the year preceding the pregnancy, representing $9.1 \%$ of all women. Age appeared to be a significant risk factor in this case $(p=.05)$ as women in their thirties were more likely to be exposed to physical abuse before pregnancy. Moreover, having less than four children $(p=$ $.02)$ and lower education $(p=.03)$ appeared to be significant risk factors.

A total of 72 women were physically abused in pregnancy, representing $17.1 \%$ of all abused women. A quarter of them were physically abused for the first time during pregnancy. This can be compared with the 121 women who were abused before pregnancy, who represent $28.8 \%$ of all abused women. Among these women, $55.4 \%$ did not report abuse during pregnancy.

A total of 37 women were physically, emotionally, and sexually abused during pregnancy, 
Table 2: Sociodemographic characteristics and form of abuse during pregnancy for survey participants reporting and not reporting violence during pregnancy $(N=1,330)^{a}$

\begin{tabular}{|c|c|c|c|c|c|c|c|c|c|}
\hline \multirow[b]{2}{*}{ Variable } & \multicolumn{3}{|c|}{ Emotional abuse } & \multicolumn{3}{|c|}{ Physical abuse } & \multicolumn{3}{|c|}{ Sexual abuse } \\
\hline & Yes & No & $p$ & Yes & No & $p$ & Yes & No & $p$ \\
\hline Age, years & $n=345$ & $n=985$ & & $n=72$ & $n=1,258$ & & $n=180$ & $n=1,150$ & \\
\hline $14-19, n=32$ & $\begin{array}{c}5 \\
15.6 \% \\
1.4 \%\end{array}$ & $\begin{array}{c}27 \\
84.4 \% \\
2.7 \%\end{array}$ & .143 & $\begin{array}{c}2 \\
6.3 \% \\
2.8 \%\end{array}$ & $\begin{array}{c}30 \\
93.8 \% \\
2.4 \%\end{array}$ & .718 & $\begin{array}{c}9 \\
28.1 \% \\
5.0 \%\end{array}$ & $\begin{array}{c}23 \\
71.9 \% \\
2.0 \%\end{array}$ & .136 \\
\hline $20-29, n=660$ & $\begin{array}{c}156 \\
23.6 \% \\
45.2 \%\end{array}$ & $\begin{array}{c}504 \\
76.4 \% \\
51.2 \%\end{array}$ & & $\begin{array}{c}32 \\
4.8 \% \\
44.4 \%\end{array}$ & $\begin{array}{c}628 \\
95.2 \% \\
49.9 \%\end{array}$ & & $\begin{array}{c}83 \\
12.6 \% \\
46.1 \%\end{array}$ & $\begin{array}{c}577 \\
87.4 \% \\
50.2 \%\end{array}$ & \\
\hline $30-39, n=512$ & $\begin{array}{c}147 \\
28.7 \% \\
42.6 \%\end{array}$ & $\begin{array}{c}365 \\
71.3 \% \\
37.1 \%\end{array}$ & & $\begin{array}{c}33 \\
6.4 \% \\
45.8 \%\end{array}$ & $\begin{array}{c}479 \\
93.6 \% \\
38.1 \%\end{array}$ & & $\begin{array}{c}69 \\
13.5 \% \\
38.3 \%\end{array}$ & $\begin{array}{c}443 \\
86.5 \% \\
38.5 \%\end{array}$ & \\
\hline $40-50, n=126$ & $\begin{array}{c}37 \\
29.0 \% \\
10.7 \%\end{array}$ & $\begin{array}{c}89 \\
71.0 \% \\
9.0 \%\end{array}$ & & $\begin{array}{c}5 \\
4.0 \% \\
6.9 \%\end{array}$ & $\begin{array}{c}121 \\
96.0 \% \\
9.7 \%\end{array}$ & & $\begin{array}{c}19 \\
15.3 \% \\
10.6 \%\end{array}$ & $\begin{array}{c}107 \\
84.7 \% \\
9.3 \%\end{array}$ & \\
\hline Number of children & $n=345$ & $n=985$ & & $n=72$ & $n=1,258$ & & $n=180$ & $n=1,150$ & \\
\hline $0, n=254$ & $\begin{array}{c}43 \\
16.9 \% \\
12.5 \%\end{array}$ & $\begin{array}{c}211 \\
83.1 \% \\
21.4 \%\end{array}$ & .001 & $\begin{array}{c}7 \\
2.8 \% \\
9.7 \%\end{array}$ & $\begin{array}{c}247 \\
97.2 \% \\
19.6 \%\end{array}$ & .114 & $\begin{array}{c}23 \\
9.1 \% \\
12.8 \%\end{array}$ & $\begin{array}{c}231 \\
90.9 \% \\
20.1 \%\end{array}$ & .011 \\
\hline $1-3, n=720$ & $\begin{array}{c}188 \\
26.1 \% \\
54.5 \%\end{array}$ & $\begin{array}{c}532 \\
73.9 \% \\
54.0 \%\end{array}$ & & $\begin{array}{c}43 \\
6.0 \% \\
59.7 \%\end{array}$ & $\begin{array}{c}677 \\
94.0 \% \\
53.8 \%\end{array}$ & & $\begin{array}{c}95 \\
13.2 \% \\
52.8 \%\end{array}$ & $\begin{array}{c}625 \\
86.8 \% \\
54.3 \%\end{array}$ & \\
\hline $4-10, n=356$ & $\begin{array}{c}114 \\
32.0 \% \\
33.0 \%\end{array}$ & $\begin{array}{c}242 \\
68.0 \% \\
24.6 \%\end{array}$ & & $\begin{array}{c}22 \\
6.2 \% \\
30.6 \%\end{array}$ & $\begin{array}{c}334 \\
93.8 \% \\
26.6 \%\end{array}$ & & $\begin{array}{c}62 \\
17.4 \% \\
34.4 \%\end{array}$ & $\begin{array}{c}294 \\
82.6 \% \\
25.6 \%\end{array}$ & \\
\hline Education & $n=345$ & $n=985$ & & $n=72$ & $n=1,258$ & & $n=180$ & $n=1,150$ & \\
\hline Illiterate, $n=6$ & $\begin{array}{c}4 \\
66.7 \% \\
1.2 \%\end{array}$ & $\begin{array}{c}2 \\
33.3 \% \\
0.2 \%\end{array}$ & .058 & $\begin{array}{c}1 \\
16.7 \% \\
1.4 \%\end{array}$ & $\begin{array}{c}5 \\
83.3 \% \\
0.4 \%\end{array}$ & .889 & $\begin{array}{c}3 \\
50.0 \% \\
1.7 \%\end{array}$ & $\begin{array}{c}3 \\
50.0 \% \\
0.3 \%\end{array}$ & .069 \\
\hline $\begin{array}{l}\text { Elementary/ } \\
\text { middle school, } \\
n=101\end{array}$ & $\begin{array}{c}32 \\
31.7 \% \\
9.3 \%\end{array}$ & $\begin{array}{c}69 \\
68.3 \% \\
7.0 \%\end{array}$ & & $\begin{array}{c}6 \\
5.9 \% \\
8.3 \%\end{array}$ & $\begin{array}{c}95 \\
94.1 \% \\
7.6 \%\end{array}$ & & $\begin{array}{c}18 \\
17.8 \% \\
10.0 \%\end{array}$ & $\begin{array}{c}83 \\
82.2 \% \\
7.2 \%\end{array}$ & \\
\hline $\begin{array}{l}\text { High school, } \\
n=427\end{array}$ & $\begin{array}{c}123 \\
28.8 \% \\
35.7 \%\end{array}$ & $\begin{array}{c}304 \\
71.2 \% \\
30.9 \%\end{array}$ & & $\begin{array}{c}22 \\
5.2 \% \\
30.6 \%\end{array}$ & $\begin{array}{c}405 \\
94.8 \% \\
32.2 \%\end{array}$ & & $\begin{array}{c}63 \\
14.8 \% \\
35.0 \%\end{array}$ & $\begin{array}{c}364 \\
85.2 \% \\
31.7 \%\end{array}$ & \\
\hline Diploma, $n=57$ & $\begin{array}{c}12 \\
21.1 \% \\
3.5 \%\end{array}$ & $\begin{array}{c}45 \\
78.9 \% \\
4.6 \%\end{array}$ & & $\begin{array}{c}3 \\
5.3 \% \\
4.2 \%\end{array}$ & $\begin{array}{c}54 \\
94.7 \% \\
4.3 \%\end{array}$ & & $\begin{array}{c}5 \\
8.8 \% \\
2.8 \%\end{array}$ & $\begin{array}{c}52 \\
91.2 \% \\
4.5 \%\end{array}$ & \\
\hline $\begin{array}{l}\text { University, } \\
n=711\end{array}$ & $\begin{array}{c}169 \\
23.8 \% \\
49.0 \%\end{array}$ & $\begin{array}{c}542 \\
76.2 \% \\
55.0 \%\end{array}$ & & $\begin{array}{c}39 \\
5.5 \% \\
54.2 \%\end{array}$ & $\begin{array}{c}672 \\
94.5 \% \\
53.4 \%\end{array}$ & & $\begin{array}{c}89 \\
12.5 \% \\
49.4 \%\end{array}$ & $\begin{array}{c}622 \\
87.5 \% \\
54.1 \%\end{array}$ & \\
\hline Masters, $n=16$ & $\begin{array}{c}3 \\
18.8 \% \\
0.9 \%\end{array}$ & $\begin{array}{c}13 \\
81.3 \% \\
1.3 \%\end{array}$ & & $\begin{array}{c}1 \\
6.3 \% \\
1.4 \%\end{array}$ & $\begin{array}{c}15 \\
93.8 \% \\
1.2 \%\end{array}$ & & $\begin{array}{c}1 \\
6.3 \% \\
0.6 \%\end{array}$ & $\begin{array}{c}15 \\
93.8 \% \\
1.3 \%\end{array}$ & \\
\hline $\begin{array}{l}\text { Doctorate, } \\
n=12\end{array}$ & $\begin{array}{c}2 \\
16.7 \% \\
0.6 \%\end{array}$ & $\begin{array}{c}10 \\
83.3 \% \\
1.0 \%\end{array}$ & & $\begin{array}{c}0 \\
0.0 \% \\
0.0 \%\end{array}$ & $\begin{array}{c}12 \\
100.0 \% \\
1.0 \%\end{array}$ & & $\begin{array}{c}1 \\
8.3 \% \\
0.6 \%\end{array}$ & $\begin{array}{c}11 \\
91.7 \% \\
1.0 \%\end{array}$ & \\
\hline
\end{tabular}


Table 2, continued

\begin{tabular}{|c|c|c|c|c|c|c|c|c|c|}
\hline \multirow[b]{2}{*}{ Variable } & \multicolumn{3}{|c|}{ Emotional abuse } & \multicolumn{3}{|c|}{ Physical abuse } & \multicolumn{3}{|c|}{ Sexual abuse } \\
\hline & Yes & No & $p$ & Yes & No & $p$ & Yes & No & $p$ \\
\hline $\begin{array}{l}\text { Family income, } \\
\text { Saudi riyal/month }\end{array}$ & $n=345$ & $n=985$ & & $n=72$ & $n=1,258$ & & $n=180$ & $n=1,150$ & \\
\hline$<2000, n=124$ & $\begin{array}{c}43 \\
34.7 \% \\
12.5 \%\end{array}$ & $\begin{array}{c}81 \\
65.3 \% \\
8.2 \%\end{array}$ & .041 & $\begin{array}{c}9 \\
7.3 \% \\
12.5 \%\end{array}$ & $\begin{array}{c}115 \\
92.7 \% \\
9.1 \%\end{array}$ & .584 & $\begin{array}{c}18 \\
14.5 \% \\
10.0 \%\end{array}$ & $\begin{array}{c}106 \\
85.5 \% \\
44.1 \%\end{array}$ & .078 \\
\hline $\begin{array}{l}2000-8000 \\
n=601\end{array}$ & $\begin{array}{c}158 \\
26.3 \% \\
45.8 \%\end{array}$ & $\begin{array}{c}443 \\
73.7 \% \\
45.0 \%\end{array}$ & & $\begin{array}{c}33 \\
5.5 \% \\
45.8 \%\end{array}$ & $\begin{array}{c}568 \\
94.5 \% \\
45.2 \%\end{array}$ & & $\begin{array}{c}94 \\
15.6 \% \\
52.2 \%\end{array}$ & $\begin{array}{c}507 \\
9.2 \% \\
84.4 \%\end{array}$ & \\
\hline$>8000, n=605$ & $\begin{array}{c}144 \\
23.8 \% \\
41.7 \%\end{array}$ & $\begin{array}{c}461 \\
76.2 \% \\
46.8 \%\end{array}$ & & $\begin{array}{c}30 \\
5.0 \% \\
41.7 \%\end{array}$ & $\begin{array}{c}575 \\
95.0 \% \\
45.7 \%\end{array}$ & & $\begin{array}{c}68 \\
11.2 \% \\
37.8 \%\end{array}$ & $\begin{array}{c}537 \\
88.8 \% \\
46.7 \%\end{array}$ & \\
\hline Employment & $n=345$ & $n=985$ & & $n=72$ & $n=1,258$ & & $n=180$ & $n=1,150$ & \\
\hline $\begin{array}{l}\text { Employed, } \\
n=481\end{array}$ & $\begin{array}{c}131 \\
27.2 \% \\
38.0 \%\end{array}$ & $\begin{array}{c}350 \\
72.8 \% \\
35.5 \%\end{array}$ & .227 & $\begin{array}{c}29 \\
6.0 \% \\
40.3 \%\end{array}$ & $\begin{array}{c}452 \\
94.0 \% \\
35.9 \%\end{array}$ & .265 & $\begin{array}{c}79 \\
16.4 \% \\
43.9 \%\end{array}$ & $\begin{array}{c}402 \\
83.6 \% \\
35.0 \%\end{array}$ & .013 \\
\hline $\begin{array}{l}\text { Unemployed, } \\
n=849\end{array}$ & $\begin{array}{c}214 \\
25.2 \% \\
62.0 \%\end{array}$ & $\begin{array}{c}635 \\
74.8 \% \\
64.5 \%\end{array}$ & & $\begin{array}{c}43 \\
5.1 \% \\
59.7 \%\end{array}$ & $\begin{array}{c}806 \\
94.9 \% \\
64.1 \%\end{array}$ & & $\begin{array}{c}101 \\
11.9 \% \\
56.1 \%\end{array}$ & $\begin{array}{c}748 \\
88.1 \% \\
65.0 \%\end{array}$ & \\
\hline Living arrangement & $n=345$ & $n=985$ & & $n=72$ & $n=1,258$ & & $n=180$ & $n=1,150$ & \\
\hline $\begin{array}{l}\text { With spouse, } \\
n=1,000\end{array}$ & $\begin{array}{c}252 \\
25.2 \% \\
73.0 \%\end{array}$ & $\begin{array}{c}748 \\
74.8 \% \\
75.9 \%\end{array}$ & .159 & $\begin{array}{c}53 \\
5.3 \% \\
73.6 \%\end{array}$ & $\begin{array}{c}947 \\
94.7 \% \\
75.3 \%\end{array}$ & .421 & $\begin{array}{c}130 \\
13.0 \% \\
72.2 \%\end{array}$ & $\begin{array}{c}870 \\
87.0 \% \\
75.7 \%\end{array}$ & .184 \\
\hline $\begin{array}{l}\text { With spouse's } \\
\text { family, } n=330\end{array}$ & $\begin{array}{c}93 \\
28.2 \% \\
27.0 \%\end{array}$ & $\begin{array}{c}237 \\
71.8 \% \\
24.1 \%\end{array}$ & & $\begin{array}{c}19 \\
5.8 \% \\
26.4 \%\end{array}$ & $\begin{array}{c}311 \\
94.2 \% \\
24.7 \%\end{array}$ & & $\begin{array}{c}50 \\
15.2 \% \\
27.8 \%\end{array}$ & $\begin{array}{c}280 \\
84.8 \% \\
24.3 \%\end{array}$ & \\
\hline
\end{tabular}

a The first percentage given in a cell is for the number in that row. The second percentage is for the number in that column.

representing $9 \%$ of women abused in pregnancy and $2.8 \%$ of all women who participated.

Among the 420 women who were abused, $299(71.2 \%)$ women answered that they were unwilling to report the abusive acts to a medical authority. Eighty-nine (29.8\%) of the women indicated they believed that the abuse was a private matter that must be resolved internally, without seeking help outside the family framework. Fifty-seven $(19.1 \%)$ women said they thought that what had happened was an isolated incident that would not be repeated. Fifty-three (17.7\%) women indicated they believed that the husband was under stress at the time of the assault, and that it would resolve itself over time. Forty-two (14\%) women said they were afraid that reporting the assault would lead to divorce. Thirty $(10 \%)$ women indicated they thought that reporting the abuse might expose them to more assault. Twenty-four (8\%) women said they believed that the assault by the husband was justifiable because they had failed in their duties toward him. Only $15(5 \%)$ women said the assault was justifiable because of religious reasons. Moreover, $84(28.1 \%)$ women said they believed that reporting the abuse would not help them. The women's reasons are shown in Figure 1.

None of the sociodemographic variables were significantly associated with the survey participants' agreement or refusal to report abuse, as presented in Table 4. 
Table 3: Sociodemographic characteristics of survey participants abused before and during pregnancy, compared with all participants $(N=1,330)^{\text {a }}$

\begin{tabular}{|c|c|c|c|c|c|c|c|c|c|}
\hline \multirow[b]{2}{*}{ Variable } & \multicolumn{3}{|c|}{$\begin{array}{c}\text { Physical abuse } 12 \\
\text { months before pregnancy }\end{array}$} & \multicolumn{3}{|c|}{$\begin{array}{l}\text { Any type of abuse } \\
\text { during pregnancy }\end{array}$} & \multicolumn{3}{|c|}{$\begin{array}{l}\text { Any type of abuse } \\
\text { in all participants }\end{array}$} \\
\hline & Yes & No & $p$ & Yes & No & $p$ & Yes & No & $p$ \\
\hline Age, years & $n=121$ & $n=1,209$ & & $n=410$ & $n=920$ & & $n=420$ & $n=910$ & \\
\hline $14-19, n=32$ & $\begin{array}{c}2 \\
6.3 \% \\
1.7 \%\end{array}$ & $\begin{array}{c}30 \\
93.8 \% \\
2.5 \%\end{array}$ & .051 & $\begin{array}{c}9 \\
28.1 \% \\
2.2 \%\end{array}$ & $\begin{array}{c}23 \\
71.9 \% \\
2.5 \%\end{array}$ & .221 & $\begin{array}{c}9 \\
28.1 \% \\
2.1 \%\end{array}$ & $\begin{array}{c}23 \\
71.9 \% \\
2.5 \%\end{array}$ & .180 \\
\hline $20-29, n=660$ & $\begin{array}{c}51 \\
7.7 \% \\
42.1 \%\end{array}$ & $\begin{array}{c}609 \\
92.3 \% \\
50.4 \%\end{array}$ & & $\begin{array}{c}185 \\
28.0 \% \\
45.1 \%\end{array}$ & $\begin{array}{c}475 \\
72.0 \% \\
51.6 \%\end{array}$ & & $\begin{array}{c}189 \\
28.6 \% \\
45.0 \%\end{array}$ & $\begin{array}{c}472 \\
71.4 \% \\
51.8 \%\end{array}$ & \\
\hline $30-39, n=512$ & $\begin{array}{c}58 \\
11.3 \% \\
47.9 \%\end{array}$ & $\begin{array}{c}454 \\
88.7 \% \\
37.6 \%\end{array}$ & & $\begin{array}{c}171 \\
33.4 \% \\
41.7 \%\end{array}$ & $\begin{array}{c}341 \\
66.6 \% \\
37.1 \%\end{array}$ & & $\begin{array}{c}176 \\
34.4 \% \\
41.9 \%\end{array}$ & $\begin{array}{c}336 \\
65.6 \% \\
36.9 \%\end{array}$ & \\
\hline $40-45, n=126$ & $\begin{array}{c}10 \\
7.3 \% \\
8.2 \%\end{array}$ & $\begin{array}{c}116 \\
92.7 \% \\
9.6 \%\end{array}$ & & $\begin{array}{c}45 \\
35.5 \% \\
10.9 \%\end{array}$ & $\begin{array}{c}81 \\
64.5 \% \\
8.8 \%\end{array}$ & & $\begin{array}{c}46 \\
36.3 \% \\
10.9 \%\end{array}$ & $\begin{array}{c}79 \\
63.7 \% \\
8.7 \%\end{array}$ & \\
\hline Number of children & $n=121$ & $n=1,209$ & & $n=410$ & $n=920$ & & $n=420$ & $n=910$ & \\
\hline $0, n=254$ & $\begin{array}{c}14 \\
5.5 \% \\
11.6 \%\end{array}$ & $\begin{array}{c}240 \\
94.5 \% \\
19.9 \%\end{array}$ & .029 & $\begin{array}{c}52 \\
20.5 \% \\
12.7 \%\end{array}$ & $\begin{array}{c}202 \\
79.5 \% \\
22.0 \%\end{array}$ & .001 & $\begin{array}{c}53 \\
20.9 \% \\
12.6 \%\end{array}$ & $\begin{array}{c}201 \\
79.1 \% \\
22.1 \%\end{array}$ & .001 \\
\hline $1-3, n=720$ & $\begin{array}{c}65 \\
9.0 \% \\
53.7 \%\end{array}$ & $\begin{array}{c}655 \\
91.0 \% \\
54.2 \%\end{array}$ & & $\begin{array}{c}222 \\
30.8 \% \\
54.1 \%\end{array}$ & $\begin{array}{c}498 \\
69.2 \% \\
54.1 \%\end{array}$ & & $\begin{array}{c}226 \\
31.4 \% \\
53.8 \%\end{array}$ & $\begin{array}{c}494 \\
68.6 \% \\
54.3 \%\end{array}$ & \\
\hline $4-10, n=356$ & $\begin{array}{c}42 \\
11.8 \% \\
34.7 \%\end{array}$ & $\begin{array}{c}314 \\
88.2 \% \\
26.0 \%\end{array}$ & & $\begin{array}{c}136 \\
38.2 \% \\
33.2 \%\end{array}$ & $\begin{array}{c}220 \\
61.8 \% \\
23.9 \%\end{array}$ & & $\begin{array}{c}141 \\
39.6 \% \\
33.6 \%\end{array}$ & $\begin{array}{c}215 \\
60.4 \% \\
23.6 \%\end{array}$ & \\
\hline Education & $n=121$ & $n=1,209$ & & $n=410$ & $n=920$ & & $n=420$ & $n=910$ & \\
\hline Illiterate, $n=6$ & $\begin{array}{c}1 \\
16.7 \% \\
0.8 \%\end{array}$ & $\begin{array}{c}5 \\
83.3 \% \\
0.4 \%\end{array}$ & .036 & $\begin{array}{c}4 \\
66.7 \% \\
1.0 \%\end{array}$ & $\begin{array}{c}2 \\
33.3 \% \\
0.2 \%\end{array}$ & .022 & $\begin{array}{c}4 \\
66.7 \% \\
1.0 \%\end{array}$ & $\begin{array}{c}2 \\
33.3 \% \\
0.2 \%\end{array}$ & .014 \\
\hline $\begin{array}{l}\text { Elementary/ } \\
\text { middle school, } \\
n=101\end{array}$ & $\begin{array}{c}15 \\
14.9 \% \\
12.4 \%\end{array}$ & $\begin{array}{c}86 \\
85.1 \% \\
7.1 \%\end{array}$ & & $\begin{array}{c}40 \\
39.6 \% \\
9.8 \%\end{array}$ & $\begin{array}{c}61 \\
60.4 \% \\
6.6 \%\end{array}$ & & $\begin{array}{c}42 \\
41.6 \% \\
10.0 \%\end{array}$ & $\begin{array}{c}59 \\
58.4 \% \\
6.5 \%\end{array}$ & \\
\hline $\begin{array}{l}\text { High school, } \\
n=427\end{array}$ & $\begin{array}{c}50 \\
11.7 \% \\
41.3 \%\end{array}$ & $\begin{array}{c}377 \\
88.3 \% \\
31.2 \%\end{array}$ & & $\begin{array}{c}145 \\
34.0 \% \\
35.4 \%\end{array}$ & $\begin{array}{c}282 \\
66.0 \% \\
30.7 \%\end{array}$ & & $\begin{array}{c}149 \\
34.9 \% \\
35.5 \%\end{array}$ & $\begin{array}{c}278 \\
65.1 \% \\
30.5 \%\end{array}$ & \\
\hline Diploma, $n=57$ & $\begin{array}{c}2 \\
3.5 \% \\
1.7 \%\end{array}$ & $\begin{array}{c}55 \\
96.5 \% \\
4.5 \%\end{array}$ & & $\begin{array}{c}12 \\
21.1 \% \\
2.9 \%\end{array}$ & $\begin{array}{c}45 \\
78.9 \% \\
4.9 \%\end{array}$ & & $\begin{array}{c}12 \\
21.1 \% \\
2.9 \%\end{array}$ & $\begin{array}{c}45 \\
78.9 \% \\
4.9 \%\end{array}$ & \\
\hline $\begin{array}{l}\text { University, } \\
n=711\end{array}$ & $\begin{array}{c}51 \\
7.2 \% \\
42.1 \%\end{array}$ & $\begin{array}{c}660 \\
92.8 \% \\
54.6 \%\end{array}$ & & $\begin{array}{c}203 \\
28.6 \% \\
49.5 \%\end{array}$ & $\begin{array}{c}508 \\
71.4 \% \\
55.2 \%\end{array}$ & & $\begin{array}{c}206 \\
29.0 \% \\
49.0 \%\end{array}$ & $\begin{array}{c}505 \\
71.0 \% \\
55.5 \%\end{array}$ & \\
\hline Masters, $n=16$ & $\begin{array}{c}1 \\
6.3 \% \\
0.8 \%\end{array}$ & $\begin{array}{c}15 \\
93.8 \% \\
1.2 \%\end{array}$ & & $\begin{array}{c}3 \\
18.8 \% \\
0.7 \%\end{array}$ & $\begin{array}{c}13 \\
81.3 \% \\
1.4 \%\end{array}$ & & $\begin{array}{c}4 \\
25.0 \% \\
1.0 \%\end{array}$ & $\begin{array}{c}12 \\
75.0 \% \\
1.3 \%\end{array}$ & \\
\hline $\begin{array}{l}\text { Doctorate, } \\
n=12\end{array}$ & $\begin{array}{c}1 \\
8.3 \% \\
0.8 \%\end{array}$ & $\begin{array}{c}11 \\
91.7 \% \\
0.9 \%\end{array}$ & & $\begin{array}{c}3 \\
25.0 \% \\
0.7 \%\end{array}$ & $\begin{array}{c}9 \\
75.0 \% \\
1.0 \%\end{array}$ & & $\begin{array}{c}3 \\
25.0 \% \\
0.7 \%\end{array}$ & $\begin{array}{c}9 \\
75.0 \% \\
1.0 \%\end{array}$ & \\
\hline
\end{tabular}


Table 3, continued ${ }^{\mathrm{a}}$

\begin{tabular}{|c|c|c|c|c|c|c|c|c|c|}
\hline \multirow[b]{2}{*}{ Variable } & \multicolumn{3}{|c|}{$\begin{array}{c}\text { Physical abuse } 12 \\
\text { months before pregnancy }\end{array}$} & \multicolumn{3}{|c|}{$\begin{array}{l}\text { Any type of abuse } \\
\text { during pregnancy }\end{array}$} & \multicolumn{3}{|c|}{$\begin{array}{l}\text { Any type of abuse } \\
\text { in all participants }\end{array}$} \\
\hline & Yes & No & $p$ & Yes & No & $p$ & Yes & No & $p$ \\
\hline $\begin{array}{l}\text { Family income, } \\
\text { Saudi riyal/month }\end{array}$ & $n=121$ & $n=1,209$ & & $n=410$ & $n=290$ & & $n=420$ & $n=910$ & \\
\hline$<2000, n=124$ & $\begin{array}{c}15 \\
12.1 \% \\
12.4 \%\end{array}$ & $\begin{array}{c}109 \\
87.9 \% \\
9.0 \%\end{array}$ & .432 & $\begin{array}{c}45 \\
36.3 \% \\
11.0 \%\end{array}$ & $\begin{array}{c}79 \\
63.7 \% \\
8.6 \%\end{array}$ & .147 & $\begin{array}{c}45 \\
36.3 \% \\
10.7 \%\end{array}$ & $\begin{array}{c}79 \\
63.7 \% \\
8.7 \%\end{array}$ & .160 \\
\hline $\begin{array}{l}2000-8000 \\
n=601\end{array}$ & $\begin{array}{c}55 \\
9.2 \% \\
45.5 \%\end{array}$ & $\begin{array}{c}546 \\
90.8 \% \\
45.2 \%\end{array}$ & & $\begin{array}{c}193 \\
32.1 \% \\
47.1 \%\end{array}$ & $\begin{array}{c}408 \\
67.9 \% \\
44.3 \%\end{array}$ & & $\begin{array}{c}199 \\
33.1 \% \\
47.4 \%\end{array}$ & $\begin{array}{c}408 \\
66.9 \% \\
44.8 \%\end{array}$ & \\
\hline$>8000, n=605$ & $\begin{array}{c}51 \\
8.4 \% \\
42.1 \%\end{array}$ & $\begin{array}{c}554 \\
91.6 \% \\
45.8 \%\end{array}$ & & $\begin{array}{c}172 \\
28.4 \% \\
42.0 \%\end{array}$ & $\begin{array}{c}433 \\
71.6 \% \\
47.1 \%\end{array}$ & & $\begin{array}{c}176 \\
29.4 \% \\
41.9 \%\end{array}$ & $\begin{array}{c}423 \\
70.6 \% \\
46.5 \%\end{array}$ & \\
\hline Employment & $n=121$ & $n=1,209$ & & $n=410$ & $n=290$ & & $n=420$ & $n=910$ & \\
\hline $\begin{array}{l}\text { Employed, } \\
n=481\end{array}$ & $\begin{array}{c}49 \\
10.2 \% \\
40.5 \%\end{array}$ & $\begin{array}{c}432 \\
89.8 \% \\
35.7 \%\end{array}$ & .173 & $\begin{array}{c}158 \\
32.8 \% \\
38.5 \%\end{array}$ & $\begin{array}{c}323 \\
67.2 \% \\
35.1 \%\end{array}$ & .127 & $\begin{array}{c}162 \\
33.7 \% \\
38.6 \%\end{array}$ & $\begin{array}{c}319 \\
66.3 \% \\
35.1 \%\end{array}$ & .119 \\
\hline $\begin{array}{l}\text { Unemployed, } \\
n=849\end{array}$ & $\begin{array}{c}72 \\
8.5 \% \\
59.5 \%\end{array}$ & $\begin{array}{c}777 \\
91.5 \% \\
64.3 \%\end{array}$ & & $\begin{array}{c}252 \\
29.7 \% \\
61.5 \%\end{array}$ & $\begin{array}{c}597 \\
70.3 \% \\
64.9 \%\end{array}$ & & $\begin{array}{c}258 \\
30.4 \% \\
61.4 \%\end{array}$ & $\begin{array}{c}591 \\
69.6 \% \\
64.9 \%\end{array}$ & \\
\hline Living arrangement & $n=121$ & $n=1,209$ & & $n=410$ & $n=290$ & & $n=420$ & $n=910$ & \\
\hline $\begin{array}{l}\text { With spouse, } \\
n=1,000\end{array}$ & $\begin{array}{c}92 \\
9.2 \% \\
76.0 \%\end{array}$ & $\begin{array}{c}908 \\
90.8 \% \\
75.1 \%\end{array}$ & .460 & $\begin{array}{c}302 \\
30.2 \% \\
73.7 \%\end{array}$ & $\begin{array}{c}698 \\
69.8 \% \\
75.9 \%\end{array}$ & .213 & $\begin{array}{c}310 \\
31.0 \% \\
73.8 \%\end{array}$ & $\begin{array}{c}690 \\
69.0 \% \\
75.8 \%\end{array}$ & .234 \\
\hline $\begin{array}{l}\text { With spouse's } \\
\text { parents, } n=330\end{array}$ & $\begin{array}{c}29 \\
8.8 \% \\
24.0 \%\end{array}$ & $\begin{array}{c}301 \\
91.2 \% \\
24.9 \%\end{array}$ & & $\begin{array}{c}108 \\
32.7 \% \\
26.3 \%\end{array}$ & $\begin{array}{c}222 \\
67.3 \% \\
24.1 \%\end{array}$ & & $\begin{array}{c}110 \\
33.3 \% \\
26.2 \%\end{array}$ & $\begin{array}{c}220 \\
66.7 \% \\
24.2 \%\end{array}$ & \\
\hline
\end{tabular}

${ }^{a}$ The first percentage given in a cell is for the number in that row. The second percentage is for the number in that column.

Figure 1: Reasons given by survey participants for unwillingness to report abuse to a medical authority $(N=299)$

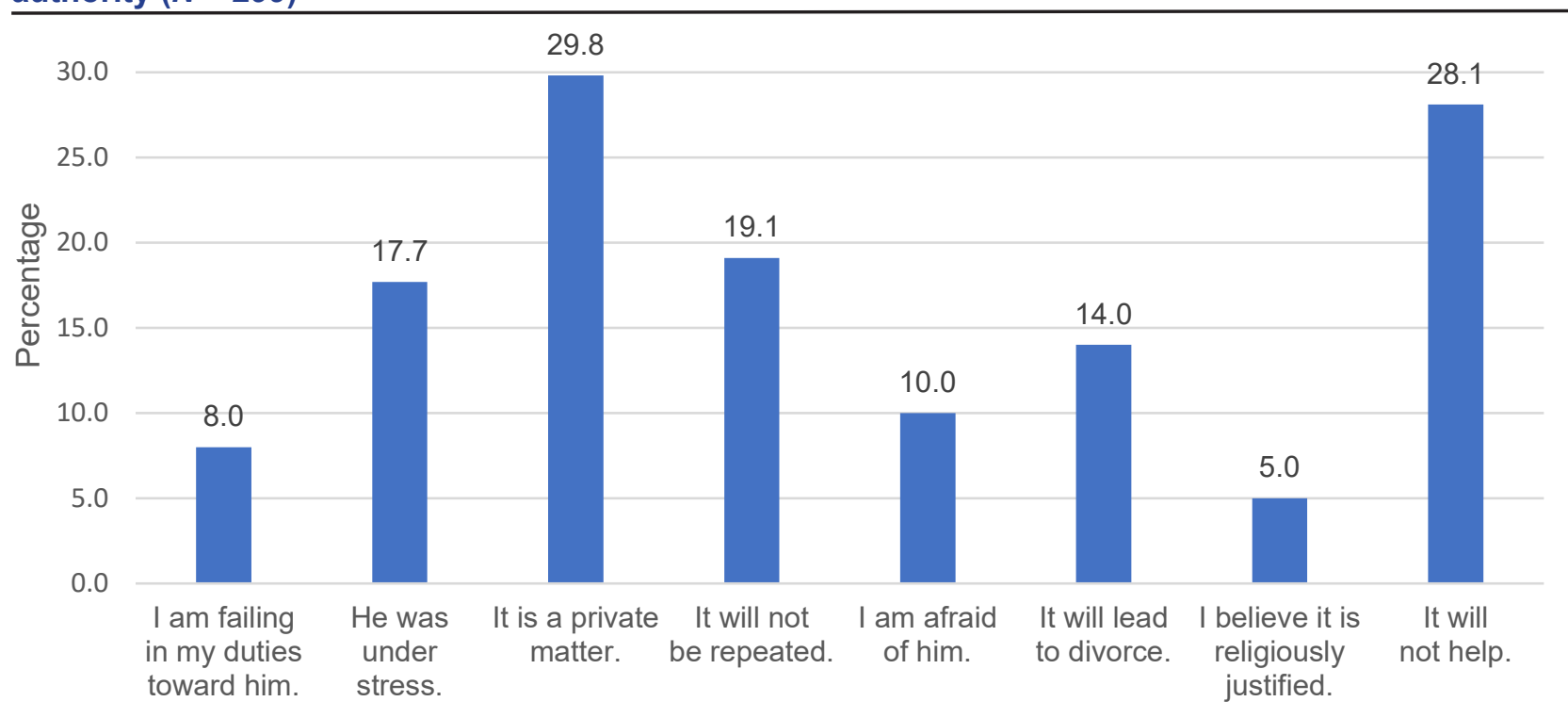

Reason 


\section{Discussion}

IPV is defined by the US Centers for Disease Control and Prevention as physical, sexual, or psychological harm by a current or former partner or spouse. In our study, we included only married women, owing to the sensitivity of asking about extramarital relationships. In our local press, cases have been reported about women killed by their ex-husbands. In 2013, a law was passed to charge husbands who harm their wives physically, sexually, or psychologically. The penalty includes a maximum sentence of three years in jail and US\$14,000 in compensation to be paid to the wife. This is only in cases in which the physical injury does not lead to permanent injury or death. In those cases, criminal law is to be applied. Before this law was passed, IPV was dealt with as a domestic problem. The issue was usually resolved between the couple, or family members may intervene to resolve the violence inflected on the woman. A small number of cases have reached a court of law, indicating that there is major underreporting of IPV, and that families may be resorting to other methods of resolution.

In a recent systemic review and metaanalysis [24], IPV during pregnancy was found to be associated with adverse infant outcomes, manifesting in preterm birth (PTB) and low birth weight (LBW). The authors included 50 articles in their final analysis. Even after adjusting for confounding factors, the pooled odds ratios for PTB and LBW were significantly increased. The effect on PTB and LBW has been associated with physical, sexual, and emotional violence. These effects have been confirmed in many other cohorts, and in cross-sectional, systemic reviews and meta-analysis [24].

Although the detrimental effects of IPV are conclusive, the prevalence of IPV
Table 4: Sociodemographic characteristics of survey participants willing and unwilling to report abuse, \% $(\mathrm{N}=1,330)^{\mathrm{a}}$

\begin{tabular}{|c|c|c|c|}
\hline Variable & $\begin{array}{c}\text { Women } \\
\text { willing to } \\
\text { report abuse }\end{array}$ & $\begin{array}{l}\text { Women } \\
\text { unwilling to } \\
\text { report abuse }\end{array}$ & $\begin{array}{c}\text { Difference, } \\
p\end{array}$ \\
\hline Age, years & $n=115$ & $n=299$ & \\
\hline $14-19, n=32$ & $\begin{array}{c}2 \\
22.2 \% \\
1.7 \%\end{array}$ & $\begin{array}{c}7 \\
77.8 \% \\
2.3 \%\end{array}$ & .172 \\
\hline $20-29, n=660$ & $\begin{array}{c}43 \\
23.1 \% \\
37.4 \%\end{array}$ & $\begin{array}{c}143 \\
76.9 \% \\
47.8 \%\end{array}$ & \\
\hline $30-39, n=512$ & $\begin{array}{c}55 \\
31.8 \% \\
47.8 \%\end{array}$ & $\begin{array}{c}118 \\
68.2 \% \\
39.5 \%\end{array}$ & \\
\hline $40-50, n=126$ & $\begin{array}{c}15 \\
31.1 \% \\
13.1 \%\end{array}$ & $\begin{array}{c}31 \\
68.9 \% \\
10.4 \%\end{array}$ & \\
\hline $\begin{array}{l}\text { Number of } \\
\text { children }\end{array}$ & $n=115$ & $n=299$ & \\
\hline $0, n=52$ & $\begin{array}{c}17 \\
32.7 \% \\
14.8 \%\end{array}$ & $\begin{array}{c}35 \\
67.3 \% \\
11.7 \%\end{array}$ & .502 \\
\hline $1-3, n=223$ & $\begin{array}{c}57 \\
25.6 \% \\
49.6 \%\end{array}$ & $\begin{array}{c}166 \\
74.4 \% \\
55.5 \%\end{array}$ & \\
\hline $4-10, n=139$ & $\begin{array}{c}41 \\
29.5 \% \\
35.7 \%\end{array}$ & $\begin{array}{c}98 \\
70.5 \% \\
32.8 \%\end{array}$ & \\
\hline Education & $n=115$ & $n=299$ & \\
\hline Illiterate, $n=4$ & $\begin{array}{c}1 \\
25.0 \% \\
0.9 \%\end{array}$ & $\begin{array}{c}3 \\
75.0 \% \\
1.0 \%\end{array}$ & .910 \\
\hline $\begin{array}{l}\text { Elementary/ } \\
\text { middle school, } \\
n=42\end{array}$ & $\begin{array}{c}12 \\
28.6 \% \\
10.4 \%\end{array}$ & $\begin{array}{c}30 \\
71.4 \% \\
10.0 \%\end{array}$ & \\
\hline $\begin{array}{l}\text { High school, } \\
n=148\end{array}$ & $\begin{array}{c}44 \\
29.7 \% \\
38.3 \%\end{array}$ & $\begin{array}{c}104 \\
70.3 \% \\
34.8 \%\end{array}$ & \\
\hline $\begin{array}{l}\text { Diploma, } \\
n=12\end{array}$ & $\begin{array}{c}5 \\
41.7 \% \\
4.3 \%\end{array}$ & $\begin{array}{c}7 \\
58.3 \% \\
2.3 \%\end{array}$ & \\
\hline $\begin{array}{l}\text { University, } \\
n=201\end{array}$ & $\begin{array}{c}51 \\
25.4 \% \\
44.3 \%\end{array}$ & $\begin{array}{c}150 \\
74.6 \% \\
50.2 \%\end{array}$ & \\
\hline Masters, $n=4$ & $\begin{array}{c}1 \\
25.0 \% \\
0.9 \%\end{array}$ & $\begin{array}{c}3 \\
75.0 \% \\
1.0 \%\end{array}$ & \\
\hline $\begin{array}{l}\text { Doctorate, } \\
n=3\end{array}$ & $\begin{array}{c}1 \\
33.3 \% \\
0.9 \%\end{array}$ & $\begin{array}{c}2 \\
66.7 \% \\
0.7 \%\end{array}$ & \\
\hline
\end{tabular}

continued 
Table 4, continued

\begin{tabular}{|c|c|c|c|}
\hline Variable & $\begin{array}{c}\text { Women } \\
\text { willing to } \\
\text { report abuse }\end{array}$ & $\begin{array}{l}\text { Women } \\
\text { unwilling to } \\
\text { report abuse }\end{array}$ & $\begin{array}{c}\text { Difference, } \\
p\end{array}$ \\
\hline $\begin{array}{l}\text { Family income, } \\
\text { Saudi riyal/month }\end{array}$ & $n=115$ & $n=299$ & \\
\hline$<2000, n=44$ & $\begin{array}{c}10 \\
22.7 \% \\
8.7 \%\end{array}$ & $\begin{array}{c}34 \\
77.3 \% \\
11.4 \%\end{array}$ & .607 \\
\hline $\begin{array}{l}2000-8000 \\
n=196\end{array}$ & $\begin{array}{c}53 \\
27.0 \% \\
46.1 \%\end{array}$ & $\begin{array}{c}143 \\
73.0 \% \\
47.8 \%\end{array}$ & \\
\hline$>8000, n=174$ & $\begin{array}{c}52 \\
29.9 \% \\
45.2 \%\end{array}$ & $\begin{array}{c}122 \\
70.1 \% \\
40.8 \%\end{array}$ & \\
\hline Employment & $\mathrm{n}=115$ & $n=299$ & \\
\hline $\begin{array}{l}\text { Employed, } \\
n=160\end{array}$ & $\begin{array}{c}49 \\
30.6 \% \\
42.6 \%\end{array}$ & $\begin{array}{c}111 \\
69.4 \% \\
37.1 \%\end{array}$ & .180 \\
\hline $\begin{array}{l}\text { Unemployed, } \\
n=254\end{array}$ & $\begin{array}{c}66 \\
26.0 \% \\
57.4 \%\end{array}$ & $\begin{array}{c}188 \\
74.0 \% \\
62.9 \%\end{array}$ & \\
\hline $\begin{array}{l}\text { Living } \\
\text { arrangement }\end{array}$ & $n=115$ & $n=299$ & \\
\hline $\begin{array}{l}\text { With spouse, } \\
n=307\end{array}$ & $\begin{array}{c}84 \\
27.4 \% \\
73.0 \%\end{array}$ & $\begin{array}{c}223 \\
72.6 \% \\
74.6 \%\end{array}$ & .419 \\
\hline $\begin{array}{l}\text { With spouse's } \\
\text { parents, } \\
n=107\end{array}$ & $\begin{array}{c}31 \\
29.0 \% \\
27.0 \%\end{array}$ & $\begin{array}{c}76 \\
71.0 \% \\
25.4 \%\end{array}$ & \\
\hline
\end{tabular}

a The first percentage given in a cell is for the number in that row. The second percentage is for the number in that column.

is variable around the globe. In some regions, there are no studies on IPV. In other regions where IPV has been addressed, the prevalence varies from as low as $0.9 \%$ in some developed countries to $70 \%$ in some developing countries. The prevalence of IPV has been estimated in one region of Saudi Arabia (Taif) to be $11.9 \%$ [25]. There are no studies in Saudi Arabia estimating the prevalence of IPV during pregnancy. In our study, the reported physical abuse during pregnancy was $5.4 \%$, which is $45 \%$ less than the general prevalence reported in Saudi Arabia. Jasinski [18] proposed that pregnancy-related factors might increase the stress experienced by the couple, which may increase the risk for IPV during pregnancy.
However, the author concluded that pregnant women were no more or less likely to be victims of IPV.

In our study, pregnancy appeared to be a protective factor against physical abuse, as the incidence of IPV decreased by more than $55 \%$. The overall prevalence of abuse in pregnancy in this study, $30.8 \%$, exceeded prevalence rates reported in some developed countries $[4,26]$, but fell within the reported range for developing counties [21]. The prevalence of psychological abuse during pregnancy in our study was $25.9 \%$, which is comparable to the prevalence rate of $26.6 \%$ in the American state of Tennessee, as reported by Gentry \& Bailey [27]. However, these prevalence rates are much higher than the prevalence of $15 \%$ reported in Malta by Debono et al. [28].

Similar to other studies, our findings revealed significant associations between IPV during pregnancy and some of the socioeconomic characteristics of the abused women. As reported by Debono et al. [28], educational status was found to be a risk factor for psychological abuse during pregnancy. In contrast, age and unemployment status were not found to be risk factors in our study. Moreover, a higher number of children and lower income were associated with higher incidence of psychological abuse during pregnancy.

Numerous studies have investigated the association between women's employment status and the risk for violence during pregnancy, with some studies finding unemployed status to be associated with an increased risk of violence and others finding no association between employment status and risk of violence [29]. Our study found that employment status is a risk factor for sexual abuse during pregnancy. However, it was not significantly associated with emotional or physical abuse.

There has been much debate about the safe and effective identification in health-care 
settings of women experiencing IPV. This study used the AAS questionnaire, a behaviourally specific tool, to estimate the prevalence of IPV among pregnant women in Saudi Arabia, to know how willing they are to seek help, and what might prevent them from seeking help.

Among the 420 abused women, 299 (71.2\%) indicated that they were unwilling to report abusive acts to a medical authority. The two most common reasons given for unwillingness to report IPV were as follows: the abuse is a private matter that must be resolved internally, without seeking help outside the family framework (29.8\%); and what happened is an isolated incident, and it will not be repeated $(19.1 \%)$. Other reasons included the belief that the husband was experiencing social stresses at the time of the assault (17.7\%), that she had failed in her duties toward her husband $(8 \%)$ and the husband was justified on religious grounds (5\%). Others were afraid that reporting the assault would lead to divorce (14\%), or that reporting the abuse might expose them to more assaults (10\%).

Another important reason for not reporting the abuse is that women thought that reporting would not help (28.1\%). This was true a decade ago, but now help is available through the Ministry of Social Affairs, either by calling and reporting the abuse through a free number (1919) or by reporting the abuse online. All reports are investigated, and a team is assembled to resolve any cases of IPV.

These findings show a high prevalence of IPV among pregnant Saudi women. This kind of adversity has been associated with maternal, fetal, and neonatal complications. As a health-care provider, we should have a holistic approach when providing care for abused women. We think the first step in addressing IPV is by raising awareness about its prevalence and effects. Women should be aware that IPV is common, and that they are not alone. Health-care providers should be trained to address IPV and to provide the necessary help through the proper agencies.
The second step is to find ways to prevent IPV by providing different approaches, such as increasing awareness, involving other family members, and better use of the Hemayah organization. Enforcing the existing law, after women report IPV to the authorities, is one factor that may prevent its recurrence. There is no simple or easy solution to such a sensitive, complex, and dangerous issue.

Conflict of Interest: none

\section{References}

1. Sohal AH, James-Hanman D. Responding to intimate partner and sexual violence against women. BMJ. 2013;346:f3100.

2. Lee D, James L, Sawires P. Preventing domestic violence: Cinical guidelines on routine screening. San Francisco, CA: Family Violence Prevention Fund; 1999.

3. Bailey B, Daugherty R. Intimate partner violence during pregnancy: Incidence and associated health behaviors in a rural population. Matern Child Health J. 2007;11(5):495-503.

4. Gazmararian J, Lazorick S, Spitz AM, Ballard T, Saltzman L, Marks JS. Prevalence of violence against pregnant women. JAMA. 1996;275(24):1915-1920.

5. Cherniak D, Grant L, Mason R, Moore B, Pellizzari, R. Intimate partner violence consensus statement. J Obstet Gynaecol Can. 2005;27(4):365-388.

6. No authors listed. ACOG Committee Opinion No. 518: Intimate partner violence. Obstet Gynecol. 2012;119(2 Pt 1):412-417.

7. Nelson HD, Bougatsos C, Blazina I. Screening women for intimate partner violence:

A systematic review to update the U.S. Preventive Services Task Force recommendation. Ann Intern Med. 2012;156(11):796-808.

8. Kiely M, El-Mohandes AAE, El-Khorazaty MN, Gantz MG. An integrated intervention to reduce intimate partner violence in pregnancy: A randomized controlled trial. Obstet Gynecol. 2010;115(2 Pt 1):273-283.

9. Bianchi AL, McFarlane J, Nava A, Gilroy H, Maddoux J, Cesario S. Rapid assessment 
to identify and quantify the risk of intimate partner violence during pregnancy. Birth. 2014;41(1):88-92.

10. Desmarais SL, Pritchard A, Lowder EM, Janssen PA. Intimate partner abuse before and during pregnancy as risk factors for postpartum mental health problems. BMC Pregnancy Childbirth. 2014;14:132.

11. Krug EG, Dahlberg LL, Mercy JA, Zwi AB, Lozano $\mathrm{R}$, eds. World report on violence and health. Geneva $(\mathrm{CH})$ : World Health Organization; 2002.

12. Murphy CC, Schei B, Myhr TL, Du Mont J. Abuse: A risk factor for low birth weight? A systematic review and meta-analysis. CMAJ. 2001;164(11):1567-1572.

13. Ganatra BR, Coyaji KJ, Rao V. Too far, too little, too late: A community-based casecontrol study of maternal mortality in rural west Maharashtra, India. Bull World Health Organ. 1998;76(6):591-598.

14. Bailey BA. Partner violence during pregnancy: Prevalence, effects, screening, and management. Int $J$ Womens Health. 2010;2:183-197.

15. Ahmed S, Koenig MA, Stephenson R. Effects of domestic violence on perinatal and early-childhood mortality: Evidence from North India. Am J Public Health (NY). 2006;96(8):1423-1428.

16. Lipsky S, Holt VL, Easterling, TR, Critchlow, $\mathrm{CW}$. Impact of police-reported intimate partner violence during pregnancy on birth outcomes. Obstet Gynecol. 2003;102(3):557-564.

17. Castro R, Peek-Asa C, Ruiz A. Violence against women in Mexico: A study of abuse before and during pregnancy. Am J Public Health (N.Y.). 2003;93(7):1110-1116.

18. Jasinski JL. Pregnancy and violence against women: An analysis of longitudinal data. $J$ Interpers Violence. 2001;16(7):712-733.

19. Sagrestano LM, Rodriguez AC, Carroll D, Bieniarz A, Greenberg A, Castro L, Nuwayhid B. A comparison of standardized measures of psychosocial variables with single-item screening measures used in an urban obstetric clinic. J Obstet Gynecol Neonatal Nurs. 2002;31(2):147-155.

20. Garcia-Moreno C, Jansen HAFM, Ellsberg M, Heise L, Watts $\mathrm{CH}$, WHO Multi-country Study on Women's Health and Domestic Violence against Women Study Team. Prevalence of intimate partner violence: Findings from the WHO Multi-country Study on Women's Health and Domestic Violence. Lancet. 2006;368(9543):1260-1269.

21. Kashif M, Murtaza K, Kirkman M. Violence against women during pregnancy in some Asian countries: A review of the literature. Ital J Public Health. 2010;7(2):6-11.

22. Shamu S, Abrahams N, Zarowsky C, Shefer $\mathrm{T}$, Temmerman M. Intimate partner violence during pregnancy in Zimbabwe: A crosssectional study of prevalence, predictors and associations with HIV. Trop Med Int Health. 2013;18(6):696-711.

23. Haj-Yahia MM. Attitudes of Arab women toward different patterns of coping with wife abuse. $J$ Interpers Violence. 2002;17(7):721-745.

24. Donovan BM, Spracklen CN, Schweizer ML, Ryckman KK, Saftlas AF. Intimate partner violence during pregnancy and the risk for adverse infant outcomes: A systematic review and meta-analysis. BJOG. 2016;123(8):1289-1299.

25. Alzahrani TA, Abaalkhail, BA, Ramadan IK. Prevalence of intimate partner violence and its associated risk factors among Saudi female patients attending the primary healthcare centers in Western Saudi Arabia. Saudi Med J. 2016;37(1):96-99.

26. Bailey BA, Daugherty RA. Intimate partner violence during pregnancy: Incidence and associated health behaviors in a rural population. Matern Child Health J. 2007;11(5):495-503.

27. Gentry J, Bailey BA. Psychological intimate partner violence during pregnancy and birth outcomes: Threat of violence versus other verbal and emotional abuse. Violence Vict. 2014;29(3):383-392.

28. Debono C, Borg Xuereb R, Scerri J, Camilleri L. Intimate partner violence: Psychological and 
verbal abuse during pregnancy. $J$ Clin Nurs. 2017;26(15-16):2426-2438.

29. Taillieu TL, Brownridge DA. Violence against pregnant women: Prevalence, patterns, risk factors, theories, and directions for future research. Aggress Violent Behav. 2010;15(1):14-35.

\section{Corresponding author}

Nourah AI Qahtani, foeto-maternal medicine, College of Medicine, King Faisal University, Dammam, King Fahd Hospital of the University, PO Box 4758, Ras Tanurah 31311, Kingdom of Saudi Arabia; nhqahtani@iau.edu.sa. 